\title{
Assistive Healthcare Robotics - Challenges in Nursing Service Innovation: Critical Review
}

\author{
Nabil Georges Badr $^{1 *}$ and Maha Dankar ${ }^{1}$ \\ ${ }^{1}$ Higher Institute of Public Health, USJ, Lebanon
}

\begin{abstract}
Healthcare robots assist older people and caregivers. Many scientists and researchers have been looking at the use of robotic technology to help not only elderly people but also their care providers. Through a review of the literature and an in-depth study of published papers, we include a timely overview of care robotics. In this study, we review what we know about the use of assistive robots in the elderly care context, their benefits and potential challenges. These humanoid resources offer a range of physical, cognitive, and social tasks in the aim of improving health outcomes. Service Science calls for understanding complex service systems, our Sociotechnical system (STS) lens applied to the use of robotics in the nursing function aims at learning the underlying trajectories of socio-technical dynamics within the nursing service system and the complexity introduced by their humanoid counterpart. This presents a significant contribution to Service Science. In essence, the research introduces the STS lens to understand the interaction between human and machine in the nursing ecosystem, while proposing learnings on a better design in practice. We focus on the current and potential future challenges of healthcare robotics as well as how such technology can help healthy aging, healthcare staff, especially nurses, and our healthcare system as a whole. We conclude that, despite the potential advantages, the adoption of care robots is still shy. Using the lens of the sociotechnical perspective and concepts of service innovation roadmaps, we pave the way for identifying factors of adoption that may influence the proliferation of care robots.
\end{abstract}

Keywords. Elderly care, nursing care robots, assistive healthcare robotics, nursing service innovation roadmaps.

\section{Introduction}

An aging population poses significant challenges to health and social care systems with limited resources. People around the world are living longer and the population growing at a rate of around $1.05 \%$ per year. The current average population increase estimates at 81 million people per year ${ }^{\dagger}$. The trend is such that, by 2050 there will be more people over 60 than under 15 , with a total population of seniors jumping to 2.1 billion up from 901 million

\footnotetext{
* Corresponding author: nabil@itvaluepartner.com

† https://www.worldometers.info/
} 
in $2015 *$ There might not be enough people to care for elderly in the future. For instance, Western Europe's population over 60 years old will increase from $21 \%$ in 2015 to $33 \%$ by 2030, while the available health worker per elderly citizen is expected to drop from 3.5 to 2.4 , which is also $30 \%$, however, in the opposite direction ${ }^{\S}$. When society's age structure shifts, a smaller number of trained caregivers and other practitioners would be required to care for a growing elderly population.

The nursing profession is responsible for the largest segment of the healthcare workforce; nurses are the cornerstone of the healthcare sector [1, 2 and 3]. The ongoing shortage of nursing professionals, combined with a high turnover of nurses and support staff at elderly-care facilities [4] has resulted in unresolved healthcare and social issues that pose significant obstacles to integrated nursing care services [5], thus affecting the safety and quality of care. Nurses have reported an increased stress due to their unmanageable workload [5], emotional fatigue, lack of motivation, and feeling of dissatisfaction [4]. In an attempt to provide technological assistance to the nursing function, one practice that is gaining momentum, is the use of robots in the care of elderly people $[6,7$, and 8$]$.

\subsection{Motivation}

The introduction of healthcare robots is starting to take center stage in assisting older adults in their autonomy and caring responsibilities as well as compensating for the lack of caregivers [9]. This phenomenon of Nursing Service innovation manifests as a service system reconfiguration, where technology occupying a major enabling function [63]. Powered by artificial intelligence robotics technology has made significant strides in recent years [10] in a variety of fields, including healthcare [14]. Robots enable elders to live independently at home and assist healthcare staff in medical facilities. Elderly care seekers, health care providers, family members, and the public have all praised assistive technologies. While the demand for care robots for the elderly and disabled is still limited, it is growing rapidly, as robot applications improve and become more user-friendly [11]. The industry forecasts that 79 million homes, globally, will have a robot in residence by $2024^{* *}$. Though impressive, the adoption of care robots in the nursing function is still relatively shy. How do assistive healthcare robots serve the nursing function? What potential benefits in use and positive outcomes could be expected? What does the literature offer on challenges and drawbacks to adoption of care robots in the nursing function? Answers to such questions may contribute to the understanding of the design principles needed for a better task-technology fit [47] at the service of the nursing function in the context of patient care.

\section{Background}

Our study is a conceptual study where we try to explore the phenomenon of the assistive technology, specifically the assistive healthcare robotics for the nursing profession in their care for older people and caregivers adopting the sociotechnical system (STS) perspective. The observation of sociotechnical factors from the perspectives of the affected stakeholders ensures that a sustainable system of interaction is created which is both engaging and

\footnotetext{
https://www.un.org/development/desa/disabilities/disability-and-ageing.html

$\S$ https://www.un.org/en/development/desa/population/publications/pdf/ageing/WorldPopulationAgeing2019Highlights.pdf

${ }^{* *}$ https://www.industryweek.com/technology-and-iiot/article/22028128/79-million-homes-globally-will-have-arobot-in-residence-by-2024
} 
benefits everyone involved [12]. Sociotechnical theory (ST) refers to the interrelatedness of social and technical aspects of an organization or a system [48]. Often relying on technology to facilitate a smart interaction with society, ST offers the tools intended to enhance the performance of work systems by recognizing the ways in which the behaviors of human actors affect the operation of technology [12].

Understanding the structure and process requirements for establishing nursing service innovation lays the foundation for safe, effective and patient-centred clinical care [64]. Through this work, we intend to underscore the importance of laying down service innovation roadmaps and responsible entities learning as we learn about potential benefits in use and positive outcome with potential hurdles that must be learned and accounted for in the Run, Transform, Innovate activities [65]. We build a contextual overview of assistive care robots, followed by a critical review of the literature to expose the sociotechnical phenomena. As we prepare for our critical review, we develop a decision plan for where to search, which terms and what sources to use and identify relevant studies, etc. We perform two searches. The first focuses our lens on different applications of assistive care robots in elderly care and the nursing function (section 2.2). Then, for the second search, we refocus on what the literature informs on benefits in use and potential challenges and drawbacks. The search encompasses online databases including but not limited to EBSCO, PubMed, Google Scholar, identifying and isolating key informative papers for our study. Then we arrange our results under themes, revealed in paragraph 3. Finally, we provide some reflection on the findings and close with suggestions for further research prospects.

\subsection{Assistive Healthcare Robotics and the Sociotechnical System Perspective}

Assistive technologies refer to technology deployed in order to augment the efficiency and effectiveness of care by predominantly supplementing the capabilities of organization and the people to perform the tasks [49]. This is the basic idea behind current developments in care robotics. The underlying theory, building on sociotechnical systems theory [50], is that the technological aspects in the design of a device will have an effect on the device operators (Fig. 1). This places demands on the operators' perceptual, cognitive, or motor capabilities, and consequently, negative outcomes can be expected, particularly under conditions of worker fatigue, which compromise human capability [51].

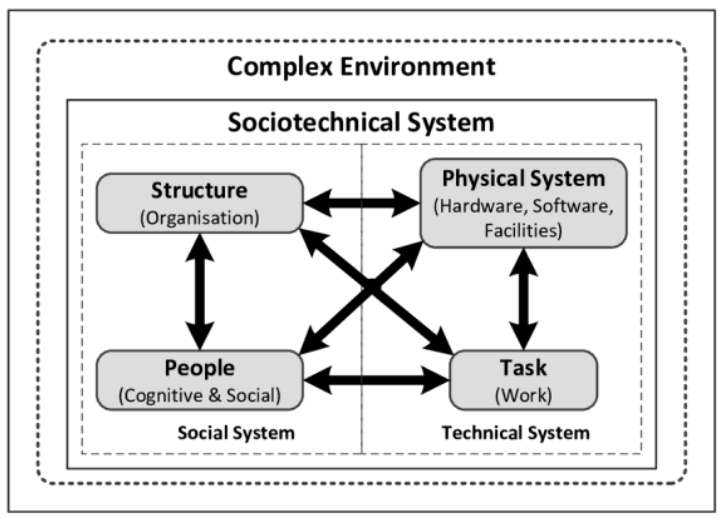

Fig. 1: Sociotechnical system (STS) - [50] 


\subsection{Elderly Assistive Care Robots Join the Nursing Job Function}

Closer to the nursing function, although there are numerous possible alternatives, ranging from better paying, valuing, and professionalizing caregiving to modern community nursing models [8], assistive technology is anticipated to play a growing role in elderly-care systems [13]. Assistive technology targets the improvement of individuals' functions and thereby promoting their wellbeing [14]. In recent years, robots have exited the operating rooms to assist in diagnosis, treatment, recovery, and nursing [5, 15, and 16]. Several models of robots that assist older people are available in a wide range of application [17].

Robots give valuable contribution to those who look after the elderly [18]. Nursing robots can execute routinely scheduled rounds, as they are not susceptible to fatigue, boredom, burnout, or forgetfulness [19], and can offer a welcome relief to the nursing staff. For instance, they alleviate healthcare workers of time or physical strain; as well provide valuable patient information [20]. Various technical innovations are born in response to the current shortage of nursing and caregiving practitioners, as well as increasing healthcare costs [21]. It has become critical to build care robotics for and by nurses [5] as robots, by definition, communicate with, and influence, their surroundings [22].

In the last decade, monitoring vital signs and facilitating communication with family and medication reminders were the most preferable tasks and applications for robots [23]. According to Lee et al, [5], the top three nursing tasks that robots could help with are "measuring/monitoring", "mobility/ activity" and "safety treatment", and the most common popular robot tasks were "detection of falls and calling for help", "lifting" and "location monitoring", while healthcare professionals preferred the use of robots in service tasks, monitoring/alarms, telemedicine, and communication. Aimed at informing the patient as well as easing the task of the care provider, in home health monitoring robots are used to track physical health status (weight, sleeping patterns, high blood pressure, and so on) using clinical and medical information. Health monitoring robots include fall detection/ prevention robots can detect and avoid falls [18]. Other examples of domotic devices are reminder robots, designed to keep older adults on track with their medication and appointments, entertainment robots provide a source of amusement, such as card playing robots that stimulate cognitive skills and stimulate memory function [18].

Assistive robots are partially or completely autonomous, that perform care-related tasks for people with physical and/or mental handicaps due to age and/or health restrictions [4, 11]. They have evolved to assist and help nursing staff, elderly people, and their families or both, in care settings when offering physical, cognitive, or emotional support [14, 22]. They have the potential to simplify everyday activities for the elderly and/or handicapped, improve quality of life by increasing autonomy, provide security [11] and improve cognitive function and depression [5]. Until now, the various forms of elderly robots have primarily assisted with everyday activities, allowing for precise real-time tracking of habits and wellbeing, as well as companionship [14]. The literature bears witness to the attention distinguishing between physically and socially assistive robots. In general, physically or surgically assistive robots account for the majority of robot use in the healthcare industry, however, physically assistive robots, do not address the rising mental health burden among the elderly [2]. Socially assistive robots maintain social contact through companionship which has a positive effect on general mental and physical wellbeing, lowering the chances of depression [24] and physically assistive robots support daily activity to improve the overall health of the elderly [18]. 


\subsubsection{Physically assistive robots}

Physically assistive robots are service robots, which assist older adults with tasks of daily life [25]. They include smart homes with a variety of features designed to assist with simple tasks so that patient can stay independent [30]. Sensory assistive robots guide the visually or auditory impaired and robotic wheelchairs for mobility assistance with manipulators for reaching and carrying, personal care, eating and drinking [18]. Targeted for recovery training and agility improvement, rehabilitation robots (e.g., Physically-Assistive Robots, PARs, as Zora) aimed at enhancing the movement performance [26, 16, and 20]. Finally, the most fun of them all, are mixed assistance robots. These are domestic chore robots designed to assist the elderly in performing simple tasks of independent living, e.g. cleaning and cooking [18].

\subsubsection{Socially assistive robots}

Socially assistive robots are companion robots implemented to improve one's psychological well-being [30]. Socially assistive robots are robotic technology systems with audio, visual, and movement capabilities. They often take the form of a pet (cat, dog, etc.) or a humanoid that can listen, talk, touch, and sense light and sound [18]. These robots capable of supporting users through in their social interaction. They provide a physical embodiment that improves likeability, commitment, motivation, adherence, and task performance, all of which are important in long-term healthcare programs. Furthermore, they help patients use physiological parameters by testing and providing feedback [16, 27, and 28]. These "Companion robots", have been linked to improved psychological status in terms of stress reduction, agitation and relaxation [25, 26, 29, and 30] with decrease in care provider burden $[8,20,29$, and 31]. For more than a decade, conversational robots have been conversing with people demonstrating levels of empathy in their encounters with humans [32]; a robotic contribution, shown to improve the overall well-being of its users [2]. Other Socially assistive robots help with autism patients and often used for telepresence make two-way contact possible between the older adults and their surroundings.

\section{$3 \quad$ Methodology for our critical review}

The purpose of this study is to review what we know about the use of assistive robots in the elderly care context, their benefits and potential challenges. We look at how assistive healthcare robotics can provide support to change this unsettled equilibrium between resources and demand on the nursing function. We review the recent literature on the use of care robots from the sociotechnical perspective, one that studies the interaction between human and machine and focuses on outcomes that may or may not be advantageous to the setting. Through the sociotechnical lens, we code thematic concepts for our discussion. This review is not exhaustive, rather directional addressing the most discussed concepts in the literature, in an attempt to learn the dynamics within the nursing service system and the complexity introduced by their humanoid counterpart. This presents a significant contribution to Service Science. In essence, the research introduces the STS lens to understand the interaction between human and machine in the nursing ecosystem, while proposing learnings on a better design in practice. 


\section{$4 \quad$ Learnings from the literature}

In the context of setting up the conversation for service innovation roadmaps, our critical review uncovered numerous learnings that relate to potential benefits in use as well as many potential challenges and drawbacks affecting the user's experience. We therefore summarize these learnings as potential benefits in use juxtaposed with challenges and drawbacks to inform the innovation roadmap for long-lasting value creation.

\subsection{Potential benefits in Use}

In general, several potential benefits of using social robots in the care of elderly have been explored extensively. Research has shown that older people's attitudes are most often optimistic and that they are happy to have a robot assist them in their everyday lives $[14,8]$ and have recognized the benefits. In addition to promoting better access to care [45, 46], cost-effectiveness, satisfaction of care, reduction in incidents of violence, physical safety, security for personal privacy and integrity, psychological benefits and decrease in care burden are among benefits of using care robots in nursing.

\subsubsection{Better access to care}

Elderly people living alone are more vulnerable to falls and accidents, and they report trouble getting health care when they need it. The use of intelligent care-providing machines extends the benefits of care in remote geographical areas and open access to specialty care services that may not be available in the patient's area [45]. For instance, people who reside in areas without a sufficient population of mental healthcare practitioners can benefit from interactive virtual human care providers [46]. Accessible anywhere and at any time, even on mobile devices, virtual care avails information about health conditions, conduct question-and-answer assessments, deliver self-care counseling and therapeutic interventions.

\subsubsection{Cost-effectiveness}

In addition to improving patient outcome and quality of care, the development of intelligent machines in healthcare has the potential to bring forth significant economic benefits for healthcare providers and the services for an aging population. These care robotic providers can help to save costs for government programs or care-assurance budgets [17]. Bringing the economy of scale to care delivery, software based intelligent machines can counteract the anticipated global cost of care, projected to exceed $\$ 6$ trillion dollars by 2030 according to a report from the World Economic Forum. In general, a robotic device can save money by reducing labor costs and performing tasks that humans may find repetitive. However, it may also increase the cost for human resources to maintain and operate with the needed capital commitment to maintain the levels of quality care [34].

\subsubsection{Satisfaction of care}

The key potential benefits of social robots in care are linked to structure (efficiency) and outcome, they have the potential to enhance both physiological and psychological factors as well as the satisfaction of the cared-for [17]. Care robots may be perceived as being immune to personal biases that human therapists may have. It has the potential to simplify 
everyday activities for the elderly and/or handicapped, improve quality of life by increasing autonomy, and provide security [11]. Robots may seem always friendly and always available. Care seekers may experience less anxiety when discussing intimate, private issues with a machine than they would with another person. Others may be more comfortable disclosing information to virtual humans, during clinical interviews and prefer to interact with virtual humans than with medical staff [35]. For instance, a virtual nurse could change its mannerisms (e.g., eye contact), speech dialect, use of common term, and other characteristics to match a given cultural group and thus develop and enhance rapport with a patient and improve overall communication.

\subsubsection{Reduction in incidents of violence}

Elderly patients in care homes are a particularly vulnerable group, subjected to a wide range of violence forms, including physical, sexual, psychological, financial, administrative, performance, and neglect. Robots have been shown to reduce the risk of physical, psychological, and sexual violence, 24 hours care a day, without tiring [31] or being irritable, and are less likely to exhibit any of the negative personality characteristics associated with human employees [8].

\subsubsection{Physical safety}

These devices can carry out logistical or surveillance tasks in addition to reacting to people in the care environment [22]. Robots may provide feedback to residents, indicating whether or not they like being treated or touched. Also, robots can be sentinels for physical safety in sensing fall risk and promoting movement performance $[26,16,20]$.

\subsubsection{Security for personal privacy and integrity}

When our freedom is threatened, some of us are more likely to consider, or even prefer, the assistance of a care robot [32]. Older adults particularly those with intellectual disabilities, need ongoing assistance with everyday activities such as toileting, showering, and dressing without feeling undignified or embarrassed (if naked) by another person assisting in his or her intimate tasks, even if that person is a nurse. They may reduce the functional burdens of coping with incontinence, wandering, and uncertainty [8]. Thus, the use of robots could be an agent of personal privacy and dignity.

\subsubsection{Psychological benefits}

Emotion recognition by machines, emotion modeling, affective user modeling, and the expression of emotions by robots and virtual agents has occupied the research agenda for decades [36]. Individually, older people tend to find robots relaxing and stimulating, resulting in a rise in social interaction. However, little is understood about the existence of socially assistive robots and their possible applications in assisting adult populations' psychological well-being. The use of robots could have direct psychological benefits for older people and their families, such as minimizing isolation and enhancing social relations and communication. Conversational robots, for instance, have displayed empathy in their encounters with humans [32], while companion robots have been linked to improved psychological status of users in terms of stress reduction, agitation and relaxation $[29,26$, $30,25,8]$. 


\subsubsection{Decrease in care burden}

Social robots have also been connected to a decrease in care provider burden [31, 29, 8, and 20]. These robots can also play a role in reducing and alleviating some of the psychological burdens on professions, especially for overburdened family members, the informal care giver who are seeing their loved ones' capacity deteriorate, causing much trauma.

\subsection{Potential challenges and drawbacks}

In order to illustrate the essential requirements for understanding and learning through service innovation, this section illustrates the justaposition of risks related to the unintended changes in direct relationship between robots and their human collabrators - here are some notions from the literature:

\subsubsection{Potential risk of injury}

Interactions with robots may be hazardous. Any malfunctions or lack of proper upkeep that may lead to accidents or injuries $[8,16]$. This issue exacerbates in cases where robots are used for purposes other than therapy, such as turning patients in beds or bathing them. Another consideration is the use of sex robots in care homes, which would necessitate additional safety steps. Failsafe mechanisms are required, that detect malfunctioning robots and alter their conduct [8].

\subsubsection{Perceived loss of control}

The use of robots in the care of elderly people puts responsibility for robot maintenance on the robots themselves. This is a textbook example of moral hazard, in which one group governs resource decisions while another bears the brunt of the benefits or burdens. A reduced quality of social relationships with individual people [8] could lead to dependency and even loss of control [20].

\subsubsection{Isolation and psychological risk}

In the literature, there has been mention of the possibility of robots promoting the feeling of isolation and reduced social interaction. The introduction of social robots could have a negative impact on the care process because they limit human interaction and autonomy. There is a significant concern that introducing robots into eldercare would result in less positive interactions, reduced human contact [29]. Concerns about personal human contact being replaced by robot-assisted activities are among the ethical concerns. If robots completely or partially replace human employment, social relationships will inevitably suffer $[8,20]$. Further, issues have been raised by the academic bodies, including the privacy issue as the risk of surveillance, the feeling of being monitored or followed with inadequate data protection, and the fear that robots would undermine capabilities and thus have negative consequences on psychological health $[15,20]$.

\subsubsection{Ethics issues, deceit and embarrassment}

Empathetic robots provide sustainable companionship for elderly people. These creature are not be subjected to mood swings, lack of patience, or exhaustion. However, robot care can also be inhumane and insensitive, and it can even be deceptive. There is an increasing 
concern that using robots to deceive elderly people is unethical, based on the premise that robots' empathy is false, if not nonexistent [29, 8, 32]. According to Bradwell et al [29], despite a frail older person loving robot pets, and possibly not distinguishing between living and non-living, relatives may believe that they are experiencing embarrassment and loss of dignity as a result of deception (although it is also possible this tension would ease upon witnessing potential quality of life benefits). These ethical issues must be carefully addressed. A structured ethics code is already a demand [37], and it could even be a deciding factor in future funding opportunities [20].

\subsubsection{Technical annoyances, cost of acquisition, and maintenance}

Lack of technical knowledge was perceived as a barrier to technology adoption [38]. The staff's intentions and facilitation of support are essential to facilitating robot use. A significant barrier to robotic system implementation is the high cost of most devices, which makes them unaffordable for both individuals and organizations. It is also uncertain if these costs will be covered by health insurance or social care services [20]. Other barriers to robotic settings listed in the literature include the potential anxiety caused by klunky or loud noises, "not being adapted" to the intended environment due to being too big, and "failing technology" in certain locations $[28,20]$.

\subsubsection{Communication disruption among care personnel}

We found instances in the literature claiming that care robots may hinder communication between nurses and patients, as well as among medical personnel, limiting the flexibility and versatility of individualized nursing services [2]. While robots can solve the negative aspects of human nature, they are devoid of the human sense of compassion, empathy and comprehension [29].

\subsubsection{Perceived impact on the nursing career}

Robots may induce behavioral changes with unfavorable consequences. The increased use of robots for older adults may create incentives for health bodies to minimize and even replace human jobs with robotic jobs, which could change the character of society [20]. Healthcare workers' attitudes about the use of assistive healthcare robotics are viewed as negative due to concerns and doubts regarding the possible substitution of their jobs and positions. Many are afraid that robots will eventually take over human employment [39]. For this reason, the fear of robots replacing doctors and nurses is a major roadblock to implementation. While there is widespread agreement that robotic systems are not intended to substitute human interaction in health and social care [20], but rather to minimize workload [2], these concerns must be taken seriously. The push to create robot professions may result in a decrease of interest in pursuing nursing as a career which will then add to shortages in professionals [8]. That said, since the majority of current nursing robot prototypes are built as assistants rather than autonomous professions, the gap in care resources will inevitably widen.

\section{$5 \quad$ Reflections and Critical Review}

In our paper's context, the social systems are the nursing bodies (in a caregiver model) and the elderly (in self-care use cases) in the healthcare ecosystem. IS research have addressed 
the relationship between the social and the technical as interactions [52] with a focus on the dynamics of interplay between the two components; such as fit, alignment, entanglement, etc. [47], and discussions of the problems experienced by users because of potential misfits.

Investigations have explored how work routines and Healthcare IT (HIT) co-evolve and interact with each other in a HIT implementation that may produce those outcomes or may hinder the expected results [33].

Other research looked into assistive technologies in the practice of medicine such as robotic surgery [53] and rehabilitation practices [54, 55]. Elsewhere, contributions have discussed principles for transition to sociotechnical ecosystems for elderly care [56], system design for disabilities [57] and user requirements for inclusive technology [58].

We found that the literature partially focused on the phenomenon and its main determinants, in caring for people with learning disabilities [59], elderly care [60], in the assistance to physical disabilities [57], chronic care [61] and promoting better caregiver interaction [62]. The healthcare workforce's embrace of technology is highly reliant on the system's dependability and, as a result, their confidence [28]. As healthcare professionals adopt healthcare robotics, the elderly would become more accepting and enabling of such devices at home [17]. It has become clear that the acceptance of robotics in care settings is affected by the behavior of the end users, "the elderly", and "the caregivers" [14].

Our work has shown that there is ample evidence where healthcare robots step in to assist in nursing care. Our critical review goes a step further to explicate how nurses and care seekers may be benefitting from and threatened by technological advances at the same time [48]. We therefore reframe the conversation to show evidence of value in use (Section 4.1) and discuss potential drawbacks, as we report on our findings relating the positive outcome of the use of care robots, in the form of value in use, and drawbacks in form unintended consequences of benefits (Section 4.2). The rapid advance of technology and the comparatively slow advance of ideas about how to organize and manage change may impede the realization of benefits (Section 4.2). Even if care robots are unable to provide true care, they may be appropriate to react to and create the illusion of care for their recipients. As long as human care is scarce, robots may assist the vulnerable and give relief to caregivers. Conversely, nurses agree that machines have disadvantages and that the physical presence of a nurse is beneficial to patients. This paper recaps that care robotics disruptions can have organizational and social consequences, some of which addressed in research studies, while others will take time and more use to manifest.

\subsection{Evidence of value in use}

Robots will find their role assisting caregivers in nursing homes, or even providing companionship to the lonely. Whilst differing viewpoints on the use of robotics in different cultures continue to exist, expectations will remain that robots can make aged-care occupations less challenging, more autonomous and affording seniors to live a safer and longer independent life in their own homes. As an alternative to living in residential aged care facilities that are equipped for supporting the health and social wellbeing of elderly individuals, many older people wish to remain in their familiar social setting at home, but some are unable to do so due to family issues, illnesses, impairments, immobility, and social limitations. Our effort revealed that intelligent care providing machines such as care robots have the potential to improve health outcomes among care seekers by customizing their care. These systems could be programmed with the knowledge and skills of diverse evidence-based practice and then deliver the most appropriate therapy or integrate different approaches based on a patient's diagnostic profile, preferences, or treatment progress. 
While their developing ability to detect, classify, and respond to the patient's (user) emotions and other stimuli can be very helpful in a therapeutic setting, care robots can also help professionals to provide quality care.

\subsection{Unintended consequences of benefits - The learnings from innovation}

Human beings are benefitting from and being threatened by technological advances at the same time [48]. Although STS theory and practice has been in the background for a while, the rapid advance of technology and the comparatively slow advance of ideas about how to organize and manage change may impede the realization of benefits.

The introduction of technology in close interaction with the human function may have outcomes that are not intended or foreseen. These outcomes may mean additional work for practitioners, changes in communication patterns and workflows, often fostering an overdependence on technology [42]. These unintended consequences can be both benefits and drawbacks, sometimes with results, contrary to what was initially intended (Table 2). Evidence of this phenomenon has been associated with technology introduction in different context, specifically in healthcare settings [43, 44].

Our review has uncovered common circumstances of unintended consequences of care robot introduction in the nursing function for elderly care (Table 2). The literature informs on the cost-effectiveness of care robots when assigned repetitive and mundane care tasks, increased satisfaction of care and reduction in incidents of violence with the potential of including logistical or surveillance for physical safety monitoring. However, inherent to their machine identity, robots may require costly regular maintenance and technological enhancement to reduce the potential of malfunction annoyances [34]. Any malfunctions or lack of proper upkeep that may lead to accidents or injuries [16] would fall on the shoulders of older adults [8].

Another example relates to the practitioner's experience where robots have been found to decrease the burden of care of the caregivers, in certain settings. They do so by taking over some of the more mundane and time-consuming tasks, however they should work totally under the supervision of health care provider to enhance the "human touch" and prevent the unethical use.

Table 2: Unintended Consequences of Care Robots - Sociotechnical Perspective (Our review)

\begin{tabular}{|l|l|l|}
\hline STS Dynamics & Benefit & Unintended Consequences \\
\hline Organization & $\begin{array}{l}\text { Essential where care workers are } \\
\text { unavailable }\end{array}$ & $\begin{array}{l}\text { Reliance on robots care hinders } \\
\text { communication among personnel }\end{array}$ \\
\hline People & $\begin{array}{l}\text { Promotes personal privacy and } \\
\text { dignity through companionship and } \\
\text { conversation. }\end{array}$ & $\begin{array}{l}\text { Potential increase in feelings of isolation } \\
\text { owing to less social interaction }\end{array}$ \\
\hline $\begin{array}{l}\text { Decrease the burden of care of the } \\
\text { caregivers }\end{array}$ & $\begin{array}{l}\text { Supervision of health care provider } \\
\text { required to prevent the unethical use }\end{array}$ \\
\hline System & $\begin{array}{l}\text { Cost-effectiveness of care robots } \\
\text { repetitive / mundane care tasks }\end{array}$ & $\begin{array}{l}\text { Require costly regular maintenance and } \\
\text { technological enhancement }\end{array}$ \\
\hline Task & $\begin{array}{l}\text { Increased safety and reduction of } \\
\text { violence due to monitoring. }\end{array}$ & $\begin{array}{l}\text { Malfunctions or lack of proper upkeep } \\
\text { lead to accidents or injuries }\end{array}$ \\
\hline
\end{tabular}

In settings where care workers are unavailable due to resource constraints, lack of training and time to provide adequate care, assistive healthcare robots are essential. In some instances, however, reliance on robotic care has hindered communication among care 
personnel [5], and ethical issues have also been identified as inhibitors to adoption and must be carefully addressed [29]. Therefore, the contextual use of these robotic assistants must complement the task of coordination and care of their settings in order to make sure that humans and humanoids work together for a better quality of care, and to reduce the effect of such unintended consequences.

\subsection{Value co-creation opportunity involving users in care robot design}

The literature has proposed frameworks that consider the role of robotics across the socioecological levels of healthcare - identifying specific challenges at each level and considerations pertaining to the design, development, and implementation of healthcare robots [40]. The attitude, ability, and doubts of healthcare professionals about the use of advanced technology can affect the use of care robots [39]. Personality traits, cognitive capacity, schooling, and community all play a role in how well care robots are accepted.

Some studies of people's attitudes toward care robots have appeared to portray pessimistic and extremely unpleasant outcomes. A thorough needs evaluation must be carried out to address these diverse viewpoints, which will ensure successful implementation. The importance of such evaluation is effectively summed up in guidelines for user incorporation in ambient assisted living projects: 'Determining individual user needs rather than merely guessing or generalizing can mean the difference between a true breakthrough for users and a cool technological advancement for the shelf'. This necessitates a detailed understanding of older people's needs and desires in terms of these devices. Although previous research has primarily focused on the acceptance of existing robots, it is critical to understand why older people embrace or oppose assistive robots and what perceptions they have of them in order to not only enhance the design of these robots but also establish successful marketing strategies. A reminder of the need to exploit concept of a Responsible Service logic (RSL) that should be infused in the design of technology assistive devices - a concern all the co-creators of the service, although they have different skills, activities, competencies, and even belong to different entities [66]. Users should be more involved in the development process and undergo adequate training and expertise before implementation. Different stakeholders with very different needs can interact with robotic systems in health and social care. If the real results do not fulfill the users' expectations, it is difficult to introduce such intrusively disruptive inventions. To overcome this obstacle, early conversations about potential roles and shortcomings should begin, and an iterative process should be used to include individual experiences and moderate expectations.

\subsection{Care robots, viable actors in optimizing health system performance}

Virtual humans and robots are being improved in their capability to recognize, respond to, and express emotions. Robots may also be capable of sensitivity and adaptation to specific aspects of a patient's culture such as race/ethnicity or socioeconomic status. Intelligent care providing machines, combining technologies in sensing, artificial intelligence and emotive computing, have the potential to greatly improve health outcomes among care seekers by customizing their care. These systems could be programmed with the knowledge and skills of diverse evidence-based approaches and then deliver the most appropriate therapy or integrate different approaches based on a patient's diagnostic profile, preferences, or treatment progress. Intelligent care-providing machines may also be capable of sensitivity and adaptation to specific aspects of a patient's culture such as race/ethnicity or socioeconomic status. However, the integration of AI, robotics and smart machines, is yet to fully compete with the warmth of human presence. The benefits of basic human contact, such as shaking hands before and after a session with a patient, placing a hand on 
the shoulder of a person who is overcome with grief, or handing a patient a tissue to dry their tears, are still irreplaceable.

Yet, care robots can be seen as viable actors in optimizing health system performance. Intelligent machines offer several advantages at the service of the patients and their healthcare professionals. Modern expert systems and other intelligent machines can help with highly complex tasks and do so with greater efficiency, accuracy, and reliability. Hospital robots can execute routinely scheduled rounds, they are not susceptible to fatigue, boredom, burnout, or forgetfulness [19]. Nevertheless, the use of care robots do serve the quadruple aim of care, health, cost and meaning in work [41], which emphasizes that the care of the patient requires care of the provider. This underscores importance of setting up Service Innovation Roadmaps that incorporate closed loop learning processes, to align people to the path value realization. These roadmaps would incorporate Run, Transform, Innovate activities that are essential to the cocreation of value while focused on the tactical development of better measurements and tools for understanding the past and future identities, reputations, values, goals, and strategies of entities interacting to achieve outcomes of the nursing function [65].

\section{Conclusion and further research prospects}

For future work, the literature review may extend to additional sources for example in systems thinking and robot to human interaction, taking into account Future research perspectives with link to STS approach, also taking into account contextual and cultural factors. We do however provide a valuable recommendations for further research and translate the quadruple aim for health improvement, in the context of the nursing function, where assistive robots help enhance patient experience, improve the health and life of the population while maintaining a focus on improving the work life of health care providers.

This study brings to bear practical implications of the sociotechnical dimensions of robotics. Because robotics is viewed as part of the next possible Kondratiev wave (with biotechnology), more advancements in robotics are likely as humanoid innovations launch technological revolutions that in turn create leading industrial or commercial sectors. Early implementation in this field is advantageous because the potential benefits for healthcare are enormous. In closing, care professionals understand that resistance to adopting welfare technology in general, stems from organizational, societal, technical, and ethical concerns. Sociotechnical principles of technology adoption inform that individual acceptance is essential for robotic systems to be adopted in real-life scenarios. Because it might be difficult to accept disruptive innovations, user participation is an important antecedent to the acceptance of assistive robots. In future research, it would be interesting to see how much such interaction with a robot improve acceptance.

While there is still low demand for assistive healthcare robots for the elderly or disabled, the segment is increasingly growing as robot care applications expand and become more user-friendly. The truth of the matter is that the technology's maturity and readiness are still unclear. On the adoption scope, elderly clients, health care providers, and family members have all praised assistive devices, but more research into their outcomes and efficacy is required. Future research should look at whether these problems will continue to persist or whether new technologies will better fix user interface and safety perception. User participation studies will help guide principles of usability (fit for use) and usefulness (fit for purpose) of care robots. Here too, we sense that additional research on the established environmental barriers, such as overall noise levels or spatial arrangement would be useful. Through the academic lens of this review, we discovered some important gaps in priority issues, specifically for ethical use of companion robots with older adults between the robot ethics community and real-world stakeholders. It is apparent that ethical 
and social considerations contribute to resistance in use of care robots in elder care. The subject of ethics is a touchy topic that has elicited both negative and positive reactions. We therefore recognize a need for ethical studies that consider the consequences to stakeholders and the apparent lack of agreement on commonly discussed issues. Robot care can also be perceived as inhumane and insensitive, and it can even be deceptive. Looking through an ethical lens, assistive healthcare robots are not perceived as a replacement for human care, and they should be used under the guidance of caregivers to maintain the dignity of the elderly.

Will simulated acts of empathy and benevolent kindness be experienced as analogous to the real thing? Would science reach a point where data and decision making surpass the need for the human touch? How would this affect the quality of mental health? These issues require further study, as we continue to develop intelligent care machines.

\section{References}

1. Mitzner, T. L., Chen, T. L., Kemp, C. C., \& Rogers, W. A.. Identifying the Potential for Robotics to Assist Older Adults in Different Living Environments. Int J Soc Robot, 6(2), 213-227 (2014).

2. Abdi, J., Al-Hindawi, A., Ng, T., \& Vizcaychipi, M. P. Scoping review on the use of socially assistive robot technology in elderly care. BMJ Open, 8, 1-20 (2018).

3. Christoforou, E. G., Panayides, A. S., Avgousti, S., Masouras, P., \& Pattichis, C. S. An Overview of Assistive Robotics and Technologies for Elderly Care. In: Henriques J., Neves N., de Carvalho P. (eds) XV Mediterranean Conference on Medical and Biological Engineering and Computing - MEDICON 2019, 76, 971-976. (2020)

4. Kyrarini, M., Lygerakis, F., Rajavenkatanarayanan, A., Sevastopoulos, C., Nambiappan, H. R., Chaitanya, K. K., Badu, A. R., Mathew, J., \& Makedon, F. A Survey of Robots in Healthcare. Technologies, 9, 8, 1-26. (2021)

5. Lee, J-Y., Song, Y. A., Jung, J. Y., Kim, H. J., Kim, B. R., Do, H-K., \& Lim, J-Y. Nurses' needs for care robots in integrated nursing care services. J Adv Nurs, 74, 2094-2105. (2018)

6. Broadbent, E., Garett, J., Jepsen, N., Ogilvie, V. L., Ahn, H. S., Robinson, H., Peri, K., Kerse, N., Rouse, P., Pillai, A., \& Macdonald, B. Using Robots at Home to Support Patients With Chronic Obstructive Pulmonary Disease: Pilot Randomized Controlled Trial. JMIR, 20(2), 1-15 (2018).

7. Kim, J. Use of Robots as a Creative Approach in Healthcare ICT. Health Informatics Research, 24(3), 155-156 (2018).

8. O'Brolcha' in, F. Robots and people with dementia: Unintended consequences and moral hazard. Nursing Ethics, 26(4), 962-972 (2019).

9. Hosseini, S. H., \& Goher, K. M. Personal Care Robots for Older Adults: An Overview. Asian Social Science, 13(1), 11-19 (2017).

10. Pekkarinen, S., Hennala, L., Tuisku, O., Gustafsson, C., Johansson-Pajala, R-M., Thommes, K., Hoppe, J. A., \& Melkas, H. (2020). Embedding care robots into society and practice: Socio-technical considerations. Futures, 122, 1-15.

11. Melkas, H., Hennala, L., Pekkarinen, S., \& Kyrki, V. Impacts of robot implementation on care personnel and clients in elderly-care institutions. International Journal of Medical Informatics, 134, 1-6 (2020).

12. Bednar, P. M., \& Welch, C. Socio-technical perspectives on smart working: Creating meaningful and sustainable systems. Information Systems Frontiers, 1-18 (2019).

13. Tuisku, O., Pekkarinen, S., Hennala, L., \& Melkas, H. "Robots do not replace a nurse with a beating heart": The publicity around a robotic innovation in elderly care. Information Technology and People. (2018). 
14. Johansson-Pajala, R-M., \& Gustafsson, C. Significant challenges when introducing care robots in Swedish elder care. Disability and Rehabilitation: Assistive Technology, 1-13 (2020).

15. Céspedes, N., Irfan, B., Senft, E., Cifuentes, C. A., Gutierrez, L. F., Rincon-Roncancio, M., Belpaeme, T., \& Múnera, M., A Socially Assistive Robot for Long-Term Cardiac Rehabilitation in The Real Word. Frontiers in Neurorobotics, 15, 633246, 1-19 (2021).

16. Oña, E. D., Garcia-Haro, J. M., Jardón, A., \& Balaguer, C. Robotics in Health Care: Perspectives of Robot-Aided Interventions in Clinical Practice for Rehabilitation of Upper Limbs. Applied Sciences, 9, 1-27 (2019).

17. Lukasik, S., Tobis, S., Kropinska, S., \& Suwalska, A., Role of Assistive Robots in the Care of Older People: Survey Study Among Medical and Nursing Students. JMIR, 22(8), 1-10 (2020).

18. Shishehgar, M., Kerr, D., \& Blake, J. The effectiveness of various robotic technologies in assisting older adults. Health Informatics Journal, 25(3), 892-918, (2019).

19. Luxton, D. D. Recommendations for the ethical use and design of artificial intelligent care providers. Artificial Intelligence in Medicine, 62(1), 110. (2014).

20. Servaty, R., Kersten, A., Brukamp, K., Mohler, R., \& Mueller, M. Implementation of robotic devices in nursing care. Barriers and facilitators: an integrative review. BMJ Open, 10, 1-11 (2020).

21. Christoforou, E. G.,Avgousti, S., Ramdani, N., Novales, C., \& Panayides, A. S. The Upcoming Role for Nursing and Assistive Robotics: Opportunities and Challenges Ahead. Frontiers in Digital Health, 2, 585656, 1-13 (2020).

22. Pirhonen, J., Melkas, H., Laitinen, A., \& Pekkarinen, S. Could robots strengthen the sense of autonomy of older people residing in assisted living facilities? - A future-oriented study. Ethics and information Technology, 22, 151-162 (2020).

23. Alaiad, A., \& Zhou, L. The determinants of home healthcare robots adoption: An empirical investigation. International journal of medical informatics, 83(11), 825-840 (2014).

24. Fasola, J., \& Mataric', M. J. A socially assistive robot exercise coach for the elderly. Journal of Human-Robot Interact, 2(2), 3-32. (2013).

25. Hung, L., Liu, C., Woldum, E., Au-Yeung, A., Berndt, A., Wallsworth, C., Horne, N., Gregorio, M., Mann, J., \& Chaudhury, H. The benefits of and barriers to using a social robot PARO in care settings: a scoping review. BMC Geriatrics, 19, 1-10 (2019).

26. Flandorfer, P. Population Ageing and Socially Assistive Robots for Elderly Persons: The Importance of Sociodemographic Factors for User Acceptance. International Journal of Population Research, 1-14 (2012).

27. Birks, M., Bodak, M., Barlas, J., Harwood, J., \& Pether, M. (2016). Robotic Seals as Therapeutic Tools in an Aged Care Facility: A Qualitative Study. Journal of Aging Research, 2016, 1-7 (2016).

28. Céspedes, N., Raigoso, D., Múnera, M., and Cifuentes, C. A. Long-Term Social Human-Robot Interaction for Neurorehabilitation: Robots as a Tool to Support Gait Therapy in the Pandemic. Frontiers in Neurorobotics, 15, 612034, 1-12 (2021).

29. Bradwell, H. L., Winnington, R., Thill, S., \& Jones, R. B. Ethical perceptions towards real-world use of companion robots with older people and people with dementia: survey opinions among younger adults. BMC Geriatrics, 20, 1-10 (2020).

30. Hersh, M. Overcoming Barriers and Increasing Independence - Service Robots for Elderly and Disabled People. International Journal of Advanced Robotic Systems, 12, 1-33 (2015).

31. Agrigoroaie, R. M., \& Tapus, A. Developing a healthcare robot with personalized behaviors and social skills for the elderly, in International Conference on Human Robot Interaction (Christchurch) (2016). 
32. Wachsmuth, I. Robots Like Me: Challenges and Ethical Issues in Aged Care. Frontiers in Psychology, 9, 1-3 (2018).

33. Goh, J. M., Gao, G., and Agarwal, R. "Evolving Work Routines: Adaptive Routinization of Information Technology in Healthcare,” ISR (22:3), pp. 565-585 (2011).

34. Pohl, M. Robotic Systems in Healthcare with Particular Reference to Innovation in the 'Fourth Industrial Revolution' - An Ethical Challenge for Management - . Journal of International and Advanced Japanese Studies, 8, 17-33 (2016).

35. Gratch, J., Wang, N., Gerten, J., Fast, E., \& Duffy, R. Creating rapport with virtual agents. In C. Pelachaud, et al. (Eds.), Intelligent virtual agents (pp. 125-138). Springer: Berlin, Heidelberg (2007).

36. Picard, R. (1997). Affective computing. Cambridge, MA: MIT Press

37. Mansouri, N., \& Goher, K. Towards Ethical Framework for Personal Care Robots: Review and Reflection. Asian Social Science, 12(10), 152-162 (2016).

38. Vichitkraivin, P., \& Naenna, T. Factors of healthcare robot adoption by medical staff in Thai government hospitals. Health and Technology, 1-13 (2020).

39. Boumans, R., Van Meulen, F., Hindriks. K., Neerincx, M., \& Olde Rikkert, M. G. M., Robot for health data acquisition among older adults: a pilot randomised controlled crossover trial. BMJ Qual Saf, 28, 793-799 (2019).

40. Mois, G., \& Beer, J. M. The Role of Healthcare Robotics in Providing Support to Older Adults: a Socio-ecological Perspective. Current Geriatrics Reports, 9, 82-89 (2020).

41. Bodenheimer, T., \& Sinsky, C.. From triple to quadruple aim: care of the patient requires care of the provider. The Annals of Family Medicine, 12(6), 573-576 (2014).

42. Tonn, B. E., \& Stiefel, D. Anticipating the unanticipated-unintended consequences of scientific and technological purposive actions. World Futures Review, 11(1), 19-50. (2019).

43. Harrison, M. I., Koppel, R., \& Bar-Lev, S. Unintended consequences of information technologies in health care-an interactive sociotechnical analysis. JAMIA, 14(5), 542-549. (2007)

44. Campbell, E. M., Sittig, D. F., Ash, J. S., Guappone, K. P., \& Dykstra, R. H. Types of unintended consequences related to computerized provider order entry. JAMIA, 13(5), 547-556 (2006).

45. Samad-Soltani, T., Rezaei-Hachesu, P., \& Ghazisaeedi, M. Pervasive decision support systems in healthcare using intelligent robots in social media. Iranian journal of public health, 46(1), 148 (2017).

46. Scoglio, A. A., Reilly, E. D., Gorman, J. A., \& Drebing, C. E. Use of social robots in mental health and well-being research: systematic review. JMIR, 21(7), e13322 (2019).

47. Strong, D. M., and Volkoff, O. "Understanding Organization-Enterprise System Fit: A Path to Theorizing the Information Technology Artifact," MISQ (34:4), pp. 731-756 (2010).

48. Pasmore, W., Winby, S., Mohrman, S. A., \& Vanasse, R. Reflections: sociotechnical systems design and organization change. Journal of Change Management, 19(2), 67-85 (2019).

49. Krings, B. J., \& Weinberger, N. Assistant without Master? Some Conceptual Implications of Assistive Robotics in Health Care. Technologies, 6(1), 13 (2018).

50. Bostrom, R.P. \& Heinen, J.S. MIS problems and failures: A socio-technical perspective. Part I: The causes. MIS Quarterly, 1(3), pp. 17. (1977) 
51. Read, G. J., Salmon, P. M., Lenné, M. G., \& Stanton, N. A. (2015).Designing sociotechnical systems with cognitive work analysis: putting theory back into practice. Ergonomics, 58(5), 822-851

52. Sarker, S., Chatterjee, S., Xiao, X., Elbanna, A.: The Sociotechnical Axis of Cohesion for IS discipline: its historical legacy and its continued relevance. MIS Q. 43, 695-719 (2019).

53. Catchpole, K., Bisantz, A., Hallbeck, M. S., Weigl, M., Randell, R., Kossack, M., \& Anger, J. T. Human factors in robotic assisted surgery: Lessons from studies in the Wild'. Applied ergonomics, 78, 270-276 (2019).

54. Grüneberg, P. Empowering Patients in Interactive Unity with Machines: Engineering the HAL (Hybrid Assistive Limb) Robotic Rehabilitation System. In Humans and Devices in Medical Contexts (pp. 255-280). Palgrave Macmillan, Singapore (2021).

55. Kendall, E., Oh, S., Amsters, D., Whitehead, M., Hua, J., Robinson, P., .. \& Lightfoot, B. HabITec: A sociotechnical space for promoting the application of technology to rehabilitation. Societies, 9(4), 74 (2019).

56. Pekkarinen, S., Melkas, H., \& Hyypiä, M. Elderly Care and Digital Services: Toward a Sustainable Sociotechnical Transition. In Human-Centered Digitalization and Services (pp. 259-284). Springer, Singapore (2019).

57. Blume, S., Galis, V., \& Pineda, A. V. Introduction: STS and disability. Science, Technology, \& Human Values, 39(1), 98-104 (2014).

58. Jovanović, M., De Angeli, A., McNeill, A., \& Coventry, L. User requirements for inclusive technology for older adults. International Journal of Human-Computer Interaction, 1-19 (2021).

59. Badr, N. G., \& Asmar, M. K. Meta Principles of Technology Accessibility Design for Users with Learning Disabilities: Towards Inclusion of the Differently Enabled. In Exploring Digital Ecosystems (pp. 195-209). Springer, Cham (2020).

60. Pekkarinen, S., \& Melkas, H. Welfare state transition in the making: Focus on the niche-regime interaction in Finnish elderly care services. Technological Forecasting and Social Change, 145, 240-253 (2019).

61. Tan, S. Y., \& Taeihagh, A. Governing the adoption of robotics and autonomous systems in long-term care in Singapore. Policy and society, 1-21 (2020).

62. Badr, N. G., Sorrentino, M., \& De Marco, M. Health information technology and caregiver interaction: building healthy ecosystems. In International Conference on Exploring Service Science (pp. 316-329). Springer, Cham (2018, September).

63. P.P. Maglio, C. Breidbach, A Service Science Perspective on the Role of ICT in Service Innovation Conference: European Conference on Information Systems (ECIS) (2015)

64. Gardner, G., Gardner, A., \& O'Connell, J. (2014). Using the Donabedian framework to examine the quality and safety of nursing service innovation. Journal of clinical nursing, 23(1-2), 145-155.

65. Spohrer J. Service innovation roadmaps and responsible entities learning. ITM Web of Conferences 38(4):01001 (2021)

66. Leonard, M. Deagoicea, M, Responsible Service Logic, ITM Web Conf. 38 (2021) 